\title{
Behandlung rheumatischer immunbedingter Nebenwirkungen bei der Krebsimmuntherapie mit Immun-Checkpoint-Inhibitoren - ist es Zeit für einen Paradigmenwechsel?
}

\author{
Katerina Chatzidionysiou ${ }^{a, b}$ Matina Liapi ${ }^{a, b}$ Georgios Tsakonas ${ }^{c, d}$ Iva Gunnarsson ${ }^{a, b}$ \\ Anca Catrina ${ }^{a, b}$ \\ a Rheumatology Unit, Karolinska University Hospital, Stockholm, Schweden; b Department of Medicine Solna, Karolinska Institute, \\ Stockholm, Schweden; 'Thoracic Oncology Center, Karolinska University Hospital, Stockholm, Schweden; ${ }^{\mathrm{d} D e p a r t m e n t}$ of Oncology \\ and Pathology, Karolinska Institute, Stockholm, Schweden
}

\section{Schlüsselwörter}

Krebsimmuntherapie · Immuncheckpoint-Inhibitoren

Immunbedingte unerwünschte Ereignisse - Therapie

\section{Zusammenfassung}

Die Immuntherapie hat die Krebsbehandlung in den letzten Jahren revolutioniert. Mehrere monoklonale Antikörper, die spezifisch für regulatorische Checkpoint-Moleküle sind, d.h. Immuncheckpoint-Inhibitoren $(|C|)$, sind zugelassen worden und werden derzeit für verschiedene Krebsarten in unterschiedlichen Behandlungslinien eingesetzt. Die Krebsimmuntherapie zielt auf die Stärkung der Immunantwort gegen Krebszellen ab. Trotz ihrer hohen Wirksamkeit stehen $\mathrm{ICl}$ mit einem neuen Spektrum von unerwünschten Ereignissen in Zusammenhang, die auf ein autoimmunes Erscheinungsbild zurückzuführen sind und häufig als immunbedingte unerwünschte Ereignisse (irAE) bezeichnet werden, wodurch der Nutzwert dieser Arzneimittel eingeschränkt wird. Diese irAE sind recht häufig und können fast jedes Organ betreffen. Der Grad der Toxizität variiert von sehr leicht bis lebensbedrohlich. Die pathophysiologischen Mechanismen, die diesen Ereignissen zugrunde liegen, sind nicht vollständig geklärt. In dieser Übersichtsarbeit fassen wir die aktuellen Erkenntnisse speziell zu den rheu-

matischen irAE zusammen und befassen uns mit aktuellen und zukünftigen Behandlungsstrategien. In den Behandlungsleitlinien wird der Einsatz von Glukokortikoiden als Erstlinientherapie weitgehend empfohlen, wenn die symptomatische Therapie nicht wirksam ist sowie bei anhaltender und/oder mittelschwerer/ schwerer Entzündung. Zielgerichtete Therapien stehen in der Behandlungspyramide weiter oben, wenn die Reaktion auf Glukokortikoide und herkömmliche, breit wirkende Immunsuppressiva unzureichend ist sowie bei schweren Formen von irAE. Präklinische Daten geben jedoch Anlass zu Bedenken hinsichtlich des potenziellen Risikos einer Beeinträchtigung der antitumoralen Wirkung. Dieses potenzielle Risiko von Glukokortikoiden könnte zusammen mit der hohen Wirksamkeit und dem potenziellen synergistischen Effekt neuerer, gezielter Immunmodulationen wie dem Tumornekrosefaktor und der Interleukin-6-Blockade einen Paradigmenwechsel unterstützen, bei dem gezieltere Behandlungen früher in der Behandlungssequenz erwogen werden.

(c) 2020 Die Autoren 


\section{Einleitung}

Die zentrale Rolle des Immunsystems bei Krebs und das Konzept der Krebsimmuntherapie sind an sich überhaupt nicht neu [1]. Es ist seit langem bekannt, dass die Immunüberwachung für die Eliminierung von Krebszellen in den Anfangsstadien der Krebsentstehung verantwortlich ist [2]. Die Bildung von Neoantigenen, d.h. Fremdantigenen auf der Oberfläche von Krebszellen, die das Ergebnis genetischer und epigenetischer Veränderungen sind, macht Krebszellen zu einem erkennbaren Ziel für die Zerstörung durch das Immunsystem. Krebszellen können jedoch Überlebensmechanismen entwickeln und der Erkennung und Zerstörung durch das Immunsystem entgehen, indem sie bei tumorspezifischen T-Zellen eine Toleranz induzieren und die T-Zell-Funktionen in der Mikroumgebung des Tumors hemmen [3].

Es wurden bzw. werden derzeit verschiedene Ansätze für die Immuntherapie bei Krebs entwickelt. Immuncheckpoint-Inhibitoren (ICI) stellen die Immunantwort gegen Tumoren wieder her. Eine Impfung mit Tumorantigenen aktiviert Effektor-Immunzellen, um neoplastische Zellen zu bekämpfen, auch wenn diese Strategie nicht sonderlich erfolgreich war [4]. Die adoptive Zelltherapie mit der Verabreichung von Immunzellen direkt an den Patienten, die Verabreichung onkolytischer Viren zur Auslösung einer systemischen Antitumoraktivität und die Zuführung von kostimulatorischen Signalen zur Förderung der T-Zell-Aktivität, z.B. durch die Verabreichung von Zytokinen zur Stimulierung des Immunsystems des Patienten (Interleukin (IL)-2, Interferon (IFN)- $\alpha$ ), sind einige weitere Beispiele für die Krebsimmuntherapie [2]. Die CAR-T-Zell-Therapie ist eine weitere vielversprechende Immuntherapie, bei der die zytotoxischen T-Lymphozyten eines Patienten durch Gentransfer zur stabilen Expression von chimären Antigenrezeptoren (CAR) angeregt werden [5].

\section{Immuncheckpoints}

Von diesen verschiedenen Strategien wird heute am häufigsten die Blockade einiger spezifischer Immuncheckpoints, z.B. von CTLA-4 (zytotoxisches T-Lymphozyten-assoziiertes Antigen 4) und von PD-1 (programmed cell death 1)/PD-L1 (programmed cell death ligand 1), eingesetzt, die als negative Regulatoren der T-Zell-Immunfunktion wirken, unter Verwendung der sogenannten Checkpoint-Inhibitoren (CPI). In den letzten Jahren wurden mehrere monoklonale Antikörper zur Hemmung dieser Ziele entwickelt und für die Behandlung von Melanomen, nichtkleinzelligem Lungenkrebs (NSCLC) und anderen Krebsarten zugelassen. Es gibt noch weitere hemmende Immuncheckpoints wie LAG-3 (lymphocyte activation gene 3), TIM-3 (T cell immunoglobulin and mucin domain 3) und VISTA (V-domain immunoglobulin suppressor of $\mathrm{T}$ cell activation). Wir werden uns auf PD-1/PD-L1 und CTLA-4 konzentrieren, da es bereits zugelassene Behandlungsformen gibt, die auf diese Immuncheckpoints abzielen.

Zur Aktivierung von T-Zellen ist mehr als ein stimulierendes Signal erforderlich. Die Bindung der Moleküle B7-1 (CD80) oder
B7-2 (CD86) auf den antigenpräsentierenden Zellen an CD28 auf den T-Zellen liefert das für die Aktivierung der T-Zellen erforderliche kostimulatorische Signal, nachdem der T-Zell-Rezeptor (TCR) an den Haupthistokompatibilitätskomplex (MHC) gebunden hat [6]. Dies führt zur Proliferation, Differenzierung und erhöhten Überlebensrate der T-Zellen. CTLA-4 ist ein CD28-Homolog mit höherer Affinität zu B7, aber im Gegensatz zu CD28 erzeugt es kein stimulierendes Signal, das zur Unterdrückung der T-Zell-Aktivierung führt [7] (Abb. 1). Die relative Menge der CD28:B7-Bindung im Vergleich zur CTLA-4:B7-Bindung bestimmt, ob eine T-Zelle aktiviert oder anergisch wird [8]. Stimulierende Signale bewirken eine Hochregulierung von CTLA-4 [9]. In einigen Fällen, z.B. bei regulatorischen T-Zellen, wird CTLA-4 jedoch konstitutiv exprimiert und trägt wesentlich zu deren immunsuppressiven Funktionen bei [10].

Ähnlich wie CTLA-4 hemmt PD-1, ein Mitglied der B7-Familie der kostimulatorischen Rezeptoren, die Aktivierung, Proliferation und das Überleben von T-Zellen durch Bindung an PD-L1 und PD-L2 [11] (Abb. 2). Obwohl sowohl PD-L1 als auch PD-L2 Liganden von PD-1 sind und beide die Effektorfunktion von T-Zellen herunterregulieren, unterscheiden sich diese beiden Liganden in mehreren Aspekten. PD-L1 wird in geringen Mengen konstitutiv exprimiert und in fast jedem Gewebe durch IFN- $\gamma$-Signale induziert. Im Gegensatz dazu ist die PD-L2-Expression hauptsächlich auf antigenpräsentierende Zellen beschränkt. PD-L2 hat eine viel stärkere Affinität zu PD-1 als PD-L1 [12]. Es gibt Unterschiede in der Expression dieser beiden Liganden in verschiedenen Tumorarten, was therapeutische Auswirkungen haben könnte [13]. PD-1 ist auch ein Marker für «erschöpfte» T-Zellen. Die T-Zell-Erschöpfung ist ein Zustand hypofunktioneller T-Zellen als Reaktion auf eine chronische Antigenbelastung, z.B. bei chronischen Infektionen und Krebs, was zu einer suboptimalen Infektions- und Tumorkontrolle führt [14]. Allerdings wurde vor kurzem die Definition der T-Zell-Erschöpfung geändert. Inzwischen ist bekannt, dass es sich bei erschöpften T-Zellen um eine heterogene Gruppe handelt, zu der auch T-Zellen gehören, die eine gewisse Effektorfunktion behalten und eine entscheidende Rolle bei der Begrenzung von Infektionen und des Tumorwachstums spielen [15].

Die Hemmung der oben genannten Immuncheckpoints führt zur Aktivierung und Vermehrung einer höheren Anzahl von Effektor-T-Zellen und verstärkt die Antitumorantwort. Die genauen Mechanismen sind jedoch noch nicht vollständig geklärt. Genauer gesagt, führt die Blockade von CTLA-4 zur Aktivierung und Proliferation von mehr T-Zell-Klonen und reduziert die durch regulatorische T-Zellen vermittelte Immunsuppression [6]. Die PD1-Blockade stellt die Aktivität von inaktiv gewordenen, gegen den Tumor gerichteten T-Zellen wieder her [6]. PD-L1 und PD-L2 werden im Vergleich zu den B7-Liganden für CTLA-4 häufiger exprimiert. Die spezifische Hemmung von PD-L1 blockiert im Gegensatz zur PD-1-Hemmung die PD-1:PD-L1-Interaktionen, während die PD-1:PD-L2-Interaktionen erhalten bleiben, da PD-1 eine höhere Affinität zu PD-L2 als zu PD-L1 aufweist [16]. Dies könnte potenzielle Auswirkungen auf ein vorteilhafteres Toxizitätsprofil der gezielten PD-L1-Hemmung haben. 


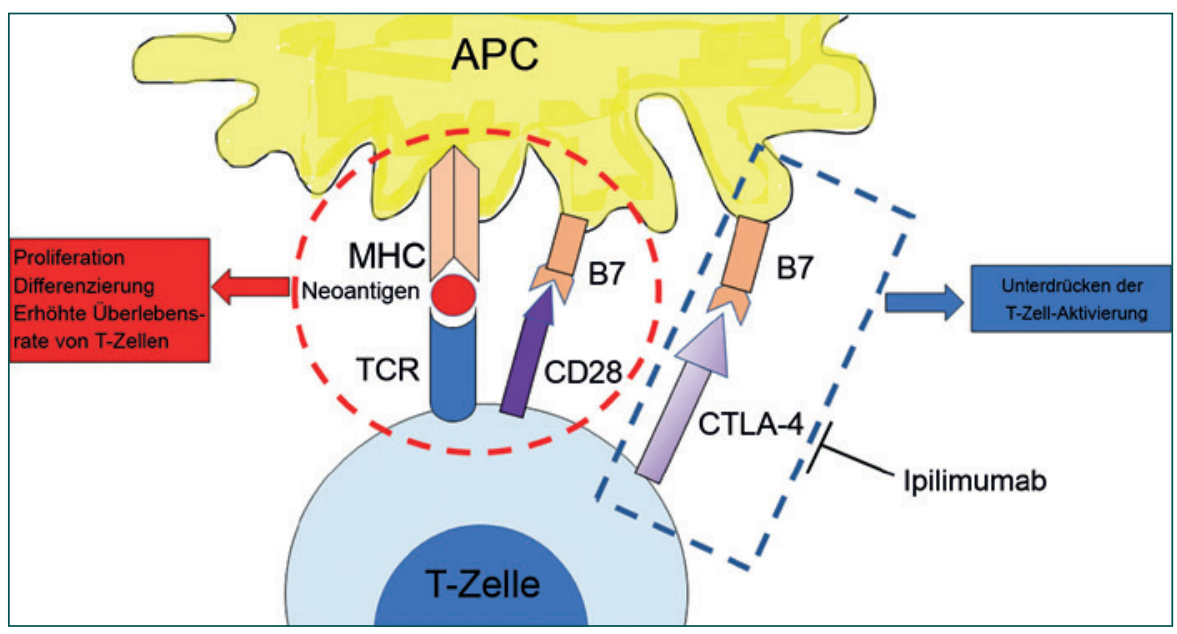

Abb. 2. Der Immuncheckpoint PD-1.

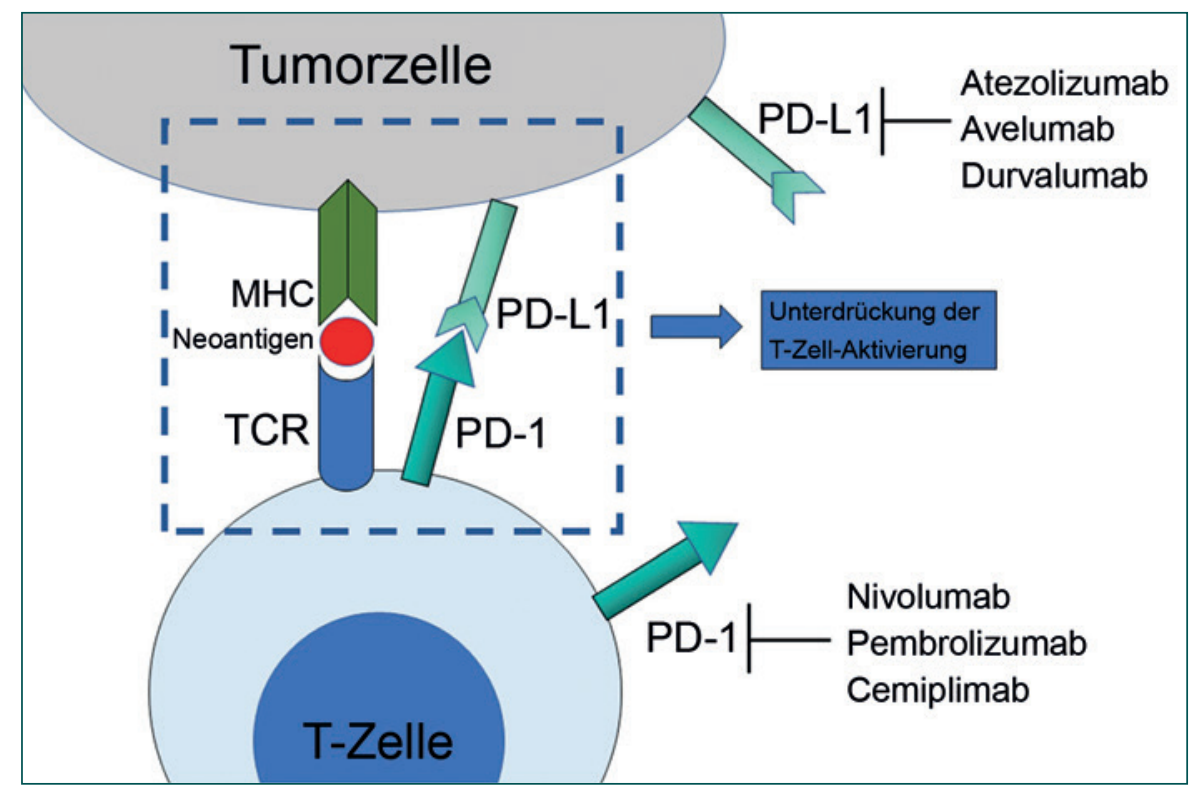

Bisher wurden zur Behandlung verschiedener solider Tumoren und hämatologischer Malignome 7 CPI von den Zulassungsbehörden zugelassen, entweder als Monotherapie oder in Kombination mit anderen Präparaten und für verschiedene Stadien der Krankheiten [17]. In Tabelle 1 sind die zugelassenen CPI, ihre Indikation, ihr Zielmolekül und der Verabreichungsweg zusammengefasst.

\section{Immunbedingte unerwünschte Ereignisse - eine neue Kategorie von Toxizität}

Da immunologische Checkpoints wichtige Regulatoren des Immunsystems sind und zur Selbsttoleranz beitragen, führt die Hemmung dieser Signalwege nicht unerwarteterweise zu einer Überaktivierung des Immunsystems und zur Entstehung von Autoimmunphänomenen. Ihre genaue Pathophysiologie wird noch nicht vollständig verstanden. Diese neue Kategorie der Toxizität, die immunbedingten unerwünschten Ereignisse (irAE), ist bemerkenswert häufig: Bei etwa 50\% der mit CPI behandelten Patienten tritt eine Form von irAE auf [18]. Ihr Schweregrad ist sehr unterschiedlich ausgeprägt und reicht von sehr leicht bis lebensbedrohlich. Sie können jedes Organsystem betreffen, entweder ein einzelnes oder mehrere Organe gleichzeitig [19]. ArnaudCoffin et al. [20] berichteten in einer systematischen Übersicht über CPI-irAE in randomisierten kontrollierten Studien (RCT) von $\mathrm{AE} \mathrm{Grad} \geq 3$ bei 14\% der mit PD-1/PD-L1-Inhibitoren behandelten Patienten, 34\% der mit CTLA-4-Inhibitoren behandelten Patienten, 55\% der Patienten, die CPI-Kombinationen erhielten, und 46\% der Patienten, die eine Immuntherapie/ChemotherapieKombination erhielten. Das Profil der irAE war je nach Behandlungskategorie unterschiedlich. Die Anwendung von CPI, insbesondere in Kombination, ist mit einer signifikanten Rate von AE des Grades $\geq 3$ verbunden. Colitis, Dermatitis, Pneumonitis und Hypophysitis sind einige Beispiele für irAE. Interessanterweise ist die Entwicklung von irAE in der Regel mit einem besseren Ansprechen auf die Behandlung verbunden [21]. 
Tab. 1. Zugelassene ICl, ihre Ziele und Indikationen

\begin{tabular}{|c|c|c|}
\hline $\begin{array}{l}\text { Monoklonaler } \\
\text { Antikörper }\end{array}$ & Ziel & Indikationen \\
\hline Ipilimumab & CTLA-4 & $\begin{array}{l}\text { Fortgeschrittenes Nierenzellkarzinom, metastasiertes kolorektales Karzinom, kutanes } \\
\text { Melanom, inoperables oder metastasiertes Melanom }\end{array}$ \\
\hline Nivolumab & PD-1 & $\begin{array}{l}\text { Metastasiertes kleinzelliges Bronchialkarzinom, inoperables oder metastasiertes Melanom, } \\
\text { lokal fortgeschrittenes oder metastasiertes Urothelkarzinom, metastasiertes kolorektales } \\
\text { Karzinom, hepatozelluläres Karzinom, metastasiertes nichtkleinzelliges Bronchialkarzinom, } \\
\text { fortgeschrittenes Nierenzellkarzinom, klassisches Hodgkin-Lymphom, rezidivierendes oder } \\
\text { metastasiertes Plattenepithelkarzinom im Kopf- und Halsbereich }\end{array}$ \\
\hline Pembrolizumab & PD-1 & $\begin{array}{l}\text { Melanom, nichtkleinzelliges Lungenkarzinom, Plattenepithelkarzinom im Kopf- und } \\
\text { Halsbereich, Hodgkin-Lymphom, Merkelzellkarzinom, hepatozelluläres Karzinom, } \\
\text { Magenkrebs, Urothelkarzinom, Gebärmutterhalskrebs }\end{array}$ \\
\hline Cemiplimab & PD-1 & Metastasiertes und lokal fortgeschrittenes Plattenepithelkarzinom der Haut \\
\hline Atezolizumab & PD-L1 & Urothelkarzinom, metastasierter nichtkleinzelliger Lungenkrebs \\
\hline Avelumab & PD-L1 & $\begin{array}{l}\text { Metastasiertes Merkelzellkarzinom, lokal fortgeschrittenes oder } \\
\text { metastasiertesUrothelkarzinom }\end{array}$ \\
\hline Durvalumab & PD-L1 & $\begin{array}{l}\text { Inoperabler nichtkleinzelliger Lungenkrebs im Stadium III, lokal fortgeschrittenes oder } \\
\text { metastasiertes Urothelkarzinom }\end{array}$ \\
\hline
\end{tabular}

Unter diesen irAE sind rheumatische Komplikationen wie Arthrose, Myositis, Sicca-Syndrom und Polymyalgia rheumatica relativ häufig und nicht nur schwierig zu diagnostizieren, sondern auch zu behandeln. Im Gegensatz zu allen anderen nichtrheumatischen irAE können rheumatologische irAE lange nach Beendigung der CPI-Behandlung auftreten und anhalten [22].

Die tatsächliche Häufigkeit von rheumatischen irAE ist nicht gut beschrieben, vor allem weil zu wenige Fälle gemeldet werden, zum Teil wegen des fehlenden Schweregrads, was zu Krankenhausaufenthalten oder zum Tod führt, aber auch, weil sie mitunter erst 2 Jahre nach der CPI-Behandlung auftreten. Darüber hinaus werden Patienten mit vorbestehenden rheumatischen Erkrankungen aufgrund einer möglichen Exazerbation der Autoimmuntoxizität von den klinischen Studien ausgeschlossen. Daher variieren die Schätzungen für die Häufigkeit rheumatischer irAE beträchtlich. Die berichtete Häufigkeit von Arthralgien/Arthritis liegt zwischen $1 \%$ und $43 \%$, die von Myalgien einschließlich des Polymyalgia rheumatica (PMR)-ähnlichen Syndroms zwischen 2\% und 20\%. Das Sicca-Syndrom tritt bei 5\% der Patienten auf, die eine Monotherapie erhalten, und bei 10\% der Patienten, die eine Kombinationstherapie erhalten [23]. Mehr als die Hälfte der mit Krebsimmuntherapien in Verbindung stehenden Vaskulitisfälle stehen im Zusammenhang mit CPI, und es wird über eine Zunahme von granulomatösen Erkrankungen (Sarkoidose), systemischer Sklerose, Lupus, Antiphospholipid-Syndrom und eosinophiler Fasziitis berichtet [23].

Ein tieferes Verständnis der Biologie der Immuncheckpoints und ihrer Hemmung ist von entscheidender Bedeutung, um optimale Behandlungsstrategien zu entwickeln. Bisher wurden mehrere Empfehlungen für das Management von irAEs veröffentlicht [2427].

\section{Management von rheumatischen irAE}

Für die Behandlung von irAE gelten einige grundlegende Prinzipien, die wir anerkennen müssen. Die therapeutischen Entscheidungen sollten in Absprache zwischen Rheumatologen, Onkologen und den Patienten getroffen werden. Die Fortsetzung oder das Absetzen der CPI sollte sich nach dem Schweregrad der irAE, der Wirksamkeit der CPI und der immunsuppressiven Behandlung richten; der interdisziplinäre Ansatz für die optimale Behandlung der Patienten ist daher entscheidend. Darüber hinaus sollte stets bedacht werden, dass ein feines Gleichgewicht angestrebt wird, bei dem der Grad der Immunsuppression hoch genug sein sollte, um die irAE zu kontrollieren, aber gleichzeitig die antitumorale Immunantwort zu erhalten.

Gemäß den kürzlich veröffentlichten Hinweisen der Europäischen Rheuma-Liga (EULAR) sollte die symptomatische Behandlung mit nichtsteroidalen Antirheumatika und/oder Analgetika die Erstbehandlung bei leichten bis mittelschweren rheumatischen irAE sein [25]. Eine lokale Behandlung mit intraartikulären Glukokortikoiden, mit oder ohne Kombination mit einer symptomatischen Behandlung, kann bei Monoarthritis oder Oligoarthritis erwogen werden. Wenn dies nicht ausreicht und die Entzündung des Gewebes weiterhin besteht, sollten systemische Glukokortikoide in Betracht gezogen werden. Einige rheumatische irAE können hohe Dosen von Glukokortikoiden erforderlich machen. In Fallberichten und Fallserien hat sich gezeigt, dass sogar bei Arthritis, die normalerweise in den meisten Fällen auf niedrige bis mittlere Dosen anspricht, hohe Dosen von Glukokortikoiden bei der Behandlung von irAE erforderlich werden können [28]. Die Identifizierung von nichtsteroidalen Behandlungsmodalitäten bei rheumatischen irAE ist daher in schweren Fällen von großer Bedeutung. 


\section{Glukokortikoide - Freund oder Feind?}

Wie oben beschrieben, stehen systemische Glukokortikoide in der Behandlungspyramide für die Kontrolle von Entzündungen bei irAE ziemlich weit oben. Es gibt jedoch nur eine begrenzte Evidenz für die Sicherheit von Glukokortikoiden, insbesondere in hohen Dosen, im Hinblick auf mögliche negative Auswirkungen auf die antitumorale Reaktion. Glukokortikoide wurden mit negativen prognostischen Eigenschaften der Immuntherapie in Verbindung gebracht, insbesondere bei Krebserkrankungen außerhalb des zentralen Nervensystems [29]. Außerdem wurde in Mausmodellen gezeigt, dass sowohl endogene als auch exogene Glukokortikoide Immunantworten gegen Krebs hemmen können [30]. In einer Studie wurde in vitro der Einfluss klinisch relevanter Dosen von Dexamethason und eines monoklonalen Anti-Tumornekrosefaktor (TNF)-Antikörpers untersucht [31]. In dieser Studie beeinträchtigten selbst niedrige Dosen von Kortikosteroiden die Antitumor-Aktivität der tumorinfiltrierenden Lymphozyten merklich. Im Gegensatz dazu hatte eine klinische Standarddosis von Infliximab, einem chimären monoklonalen Anti-TNF-Antikörper, nur eine geringe Wirkung auf die T-ZellAktivierung und die Tumorabtötung. Die Aktivität der Lymphozyten war nach Absetzen der Steroide wiederhergestellt. Exogene Glukokortikoide, die in klinisch relevanten Konzentrationen eingesetzt werden, haben auch immunsuppressive Auswirkungen auf die Fähigkeit dendritischer Zellen, Tumorantigene zu präsentieren, sowie auf die T-Zell-Aktivierung und die tumorabtötende Wirkung [32]. Diese Daten könnten darauf hindeuten, dass steroidsparende Strategien und eine frühzeitige Einleitung einer AntiTNF-Therapie für die Behandlung von irAE in der Immunonkologie in Betracht gezogen werden sollten.

Trotz dieser In-vitro-Effekte sind die klinischen Daten zur Verwendung von Kortikosteroiden widersprüchlich, obwohl derzeit nur wenige und kurze Berichte vorliegen. In einer Fallserie mit 36 Patienten mit vorbestehenden rheumatischen Erkrankungen vor der Anti-PD-1-Therapie oder mit neuen rheumatischen irAE nach der Anti-PD-1-Therapie erhielt die Mehrheit der Patienten (30 von 36) Glukokortikoide [33]. Die Wirksamkeit der Anti-PD1-Behandlung war nach wie vor hoch, insbesondere in der Melanomgruppe. Es wurden keine offensichtlichen Unterschiede in der Dauer der Kortikosteroid-Einnahme bei Patienten mit anhaltender Wirkung im Vergleich zu Patienten mit primärer oder erworbener Resistenz festgestellt. Die Daten aus einer systematischen Übersicht über die Fachliteratur ergaben, dass die gleichzeitige Verabreichung von Kortikosteroiden und ICI nicht unbedingt zu schlechteren klinischen Ergebnissen führt [34].

Es ist jedoch wichtig, darauf hinzuweisen, dass die Verabreichung von Steroiden in Dosen von mehr als 10 mg Prednisolonäquivalent zu den Ausschlusskriterien in den klinischen Studien gehörte, die zur Zulassung von ICI bei NSCLC führten [35-37]. In retrospektiven Daten von Patienten mit NSCLC, die mit ICI behandelt wurden, hat sich gezeigt, dass sich die Ansprechraten, das progressionsfreie Überleben und das Gesamtüberleben bei der Verabreichung von Steroiden in Dosen von mehr als $10 \mathrm{mg}$ Pred- nisolonäquivalent zu Beginn der Behandlung verschlechtern [38]. Eine retrospektive Studie an NSCLC-Patienten, die Steroide zur Linderung krebsbedingter Symptome erhielten, zeigte eine schlechtere Wirksamkeit von gleichzeitig verabreichten ICI [39]. Die Verabreichung von hochdosierten Steroiden $(\geq 1 \mathrm{mg} / \mathrm{kg} / \mathrm{Tag})$ ist die wichtigste Option bei der Behandlung von schweren irAE der Grade III-IV, obwohl Daten zum klinischen Ergebnis von Patienten, die aufgrund der Entwicklung von irAE Steroide erhielten, hauptsächlich aus Melanomstudien stammen. In diesen wurde berichtet, dass ihre Verabreichung keinen Einfluss auf die Wirksamkeit von ICI hat [40].

Abschließend lässt sich sagen, dass es zumindest einen theoretischen Hintergrund gibt, der auf einen negativen Einfluss der langfristigen Einnahme von Kortikosteroiden auf die Dauer der Antitumor-Reaktion hindeutet; große, prospektive Studien sind jedoch weiterhin erforderlich.

\section{Gezielte Krebstherapie - Zeit für einen Paradigmen- wechsel?}

Laut den kürzlich veröffentlichten Hinweisen sollte bei aktiven rheumatischen irAE, die eine Glukokortikoiddosis von mehr als $10 \mathrm{mg} /$ Tag Prednisolonäquivalent erforderlich machen, zunächst ein konventionelles synthetisches krankheitsmodifizierendes Antirheumatikum (csDMARD) wie Methotrexat, Hydroxychloroquin oder Sulfasalazin in Betracht gezogen werden [25]. Es gibt mehrere Fallberichte und Fallserien, die die Wirksamkeit und Sicherheit dieser Medikamente bei der Behandlung von rheumatischen irAE belegen [25]. Bei schweren irAE oder nach unzureichender Wirkung von csDMARD kann ein biologisches DMARD (bDMARD) in Betracht gezogen werden. In den letzten 2 Jahrzehnten hat sich das therapeutische Arsenal für verschiedene rheumatische Erkrankungen mit dem Aufkommen selektiver, auf das Immunsystem ausgerichteter Therapeutika, die auf pathogenetischen Prinzipien beruhen, drastisch erweitert. Zahlreiche monoklonale Antikörper und Rezeptoren, die auf entzündungsfördernde Schlüsselzytokine (wie IL-6, IL-17, IL-1, TNF) und Immunzellen (wie B-Zellen) abzielen, haben sich als wirksam erwiesen, während sich bei ihnen zudem ein akzeptables Sicherheitsprofil bei chronischen rheumatischen Erkrankungen gezeigt hat. Diese Medikamente bilden eine große Gruppe, die als biologische krankheitsmodifizierende Antirheumatika (bDMARD) bezeichnet werden (Tab. 2).

Insbesondere bei Arthritis wurde die Blockade von TNF und IL-6 erfolgreich eingesetzt. Diese beiden Zytokine spielen bei der Immunpathogenese der rheumatoiden Arthritis (RA) eine zentrale Rolle. TNF, ein sowohl für physiologische als auch für pathologische Prozesse entscheidendes Zytokin, spielt eine zentrale Rolle bei der Pathogenese zahlreicher entzündlicher Erkrankungen wie der RA und bei Morbus Crohn. Es ist auch ein wichtiger Mediator bei krebsbedingten Entzündungen [41]. Heute stehen mehrere Medikamente gegen TNF zur Verfügung, deren Wirksamkeit und Alltagswirksamkeit gut belegt sind und die ein gutes kurzund langfristiges Sicherheitsprofil aufweisen [42]. Die TNF-Hem-
Kompass Autoimmun 2022;4:2-9 DOI: $10.1159 / 000521939$ 
Tab. 2. Zugelassene biologische und zielgerichtete synthetische DMARD für die Behandlung verschiedener rheumatischer Erkrankungen

\begin{tabular}{|c|c|c|}
\hline & Ziel & Indikationen \\
\hline \multicolumn{3}{|c|}{ Biologische DMARD (bDMARD) } \\
\hline Infliximab & TNF & RA, PsA, axSpA \\
\hline Adalimumab & TNF & RA, PsA, axSpA \\
\hline Etanercept & TNF & RA, PsA, axSpA \\
\hline Certolizumab pegol & TNF & RA, PsA, axSpA \\
\hline Golimumab & TNF & RA, PsA, axSpA \\
\hline Sekukinumab & $\mid \mathrm{L}-17$ & PsA, axSpA \\
\hline Tocilizumab & IL-6R & RA, GCA \\
\hline Sarilumab & IL-6 & RA \\
\hline Abatacept & T-Zell-Kostimulation & RA, PsA \\
\hline Rituximab & CD-20 (B-Zellen) & RA, AAV, SLE* \\
\hline Belimumab & BLyS & SLE \\
\hline Anakinra & $\mathrm{IL}-1$ & RA**, Morbus Still, periodische Fiebersyndrome \\
\hline \multicolumn{3}{|c|}{ Zielgerichtete synthetische DMARD (tsDMARD) } \\
\hline Tofacitinib & JAK1/JAK3 & RA, PsA \\
\hline Baricitinib & JAK1/JAK2 & RA \\
\hline Upadacitinib & JAK1 & RA \\
\hline \multicolumn{3}{|c|}{$\begin{array}{l}\text { TNF = Tumornekrosefaktor, } \mathrm{L}=\text { = Interleukin, BLyS = B-Lymphozyten-Stimulator, JAK = Janus-Kinase, RA = rheumatoide Arthritis, PsA } \\
=\text { Psoriasis-Arthritis, axSpA = axiale Spondyloarthritis, GCA = Riesenzellarteriitis, AAV = ANCA (antineutrophile zytoplasmatische } \\
\text { Antikörper)-assoziierte Vaskulitis, SLE = systemischer Lupus erythematodes. * Off-Label-Use. }{ }^{* *} \text { Zugelassen, aber aufgrund der } \\
\text { begrenzten Wirksamkeit im Vergleich zu anderen bDMARD nicht routinemäßig eingesetzt. }\end{array}$} \\
\hline
\end{tabular}

mung wurde bei schwerer Colitis und Arthritis mit Erfolg eingesetzt [43]. Bei schwerer CPI-induzierter Colitis hat in den letzten Jahren ein Paradigmenwechsel in der Behandlung stattgefunden, wobei die TNF-Blockade die Behandlung der ersten Wahl ist und sogar als prophylaktische Maßnahme eingesetzt wird [44, 45]. Die gleichzeitige Verabreichung von ICI mit Infliximab wird derzeit in der TICIMEL-Studie (NCT03293784) untersucht. Bei Arthritis wird der Einsatz von TNF-Inhibitoren erwogen, wenn die Erstlinientherapie mit GC und csDMARD versagt hat sowie bei schweren Fällen.

IL-6 ist ein wichtiges Zytokin bei RA. Es wird von einer Vielzahl von Zellen sezerniert, unter anderem von Makrophagen, T-Zellen, B-Zellen und synovialen Fibroblasten, und gilt als übergeordnetes Zytokin im hierarchischen Zytokinnetzwerk, das an der Pathogenese der RA beteiligt ist. Es weist ein breites Spektrum an Funktionen auf, z.B. bei der B-Zell-Proliferation und Antikörperproduktion, der Hämatopoese und der T-Zell-Differenzierung $[46,47]$. Tocilizumab ist ein humanisierter monoklonaler Antikörper gegen den IL-6-Rezeptor, der zur Behandlung der aktiven RA sowohl als Monotherapie als auch in Kombination mit Methotrexat zugelassen ist. Seine Wirksamkeit und sein akzeptables Sicherheitsprofil wurden in mehreren großen randomisierten kontrollierten Studien nachgewiesen [48-51]. In einer Studie an 87 Patienten, die nach der Behandlung mit Nivolumab irAE entwickelten, wurde bei 27 von 34 Patienten, die Tocilizumab erhielten, eine klinische Verbesserung beobachtet [52]. In einer kleinen Fallserie wurde Tocilizumab erfolgreich zur Behandlung einer schweren, von CPI ausgelösten Polyarthritis eingesetzt [53].
Wie bei den Glukokortikoiden besteht auch bei der potenten zielgerichteten Therapie das Risiko einer Abschwächung der antitumoralen Wirkung der CPI, insbesondere bei langer Behandlungsdauer. Im Gegensatz zu anderen irAE, z.B. Colitis, die nach 1-maliger Verabreichung eines biologischen Wirkstoffs (z.B. eines monoklonalen Anti-TNF-Antikörpers) abklingen können, sind rheumatische irAE eher chronisch und machen zur optimalen Kontrolle der Entzündung eine langfristige Immunmodulation erforderlich. In einer kürzlich durchgeführten Studie mit einer mittleren Nachbeobachtungszeit von 9 Monaten wurde berichtet, dass die Antitumor-Reaktion bei Patienten, die mit TNF-Hemmern behandelt wurden, nicht beeinträchtigt wurde [54]. Wie bereits erwähnt, gibt es aus präklinischen Studien Beweise, die die Überlegenheit einer gezielten gegenüber einer breiteren Immunmodulation (Anti-Zytokin-Therapie gegenüber Glukokortikoiden) stützen, wobei Erstere nur einen geringen Einfluss auf die T-Zellen hat. Darüber hinaus scheint es eine potenzielle synergistische Wirkung von TNF-Hemmern mit CPI zu geben $[55,56]$.

Die gleichzeitige Behandlung von Mäusen mit TNF-Inhibitoren und einer kombinierten CTLA-4- und PD-1-Immuntherapie führte zu einer Verbesserung der Colitis und darüber hinaus zu einer verbesserten Antitumor-Wirksamkeit. Die kombinierte Blockade von IL-6- und PD-1/PD-L1-Signalen verstärkte bei Mäusen, die an einem Tumor litten, die tumorspezifische Th1Antwort und die anschließende Antitumor-Wirkung [57].

Die Daten zu anderen bDMARD wie Secukinumab, einem monoklonalen Antikörper gegen IL-17, und Rituximab, einem monoklonalen Antikörper gegen CD20, sind begrenzt. Abatacept, 
Abb. 3. Paradigmenwechsel in der Behandlung rheumatischer irAE mit CPI.

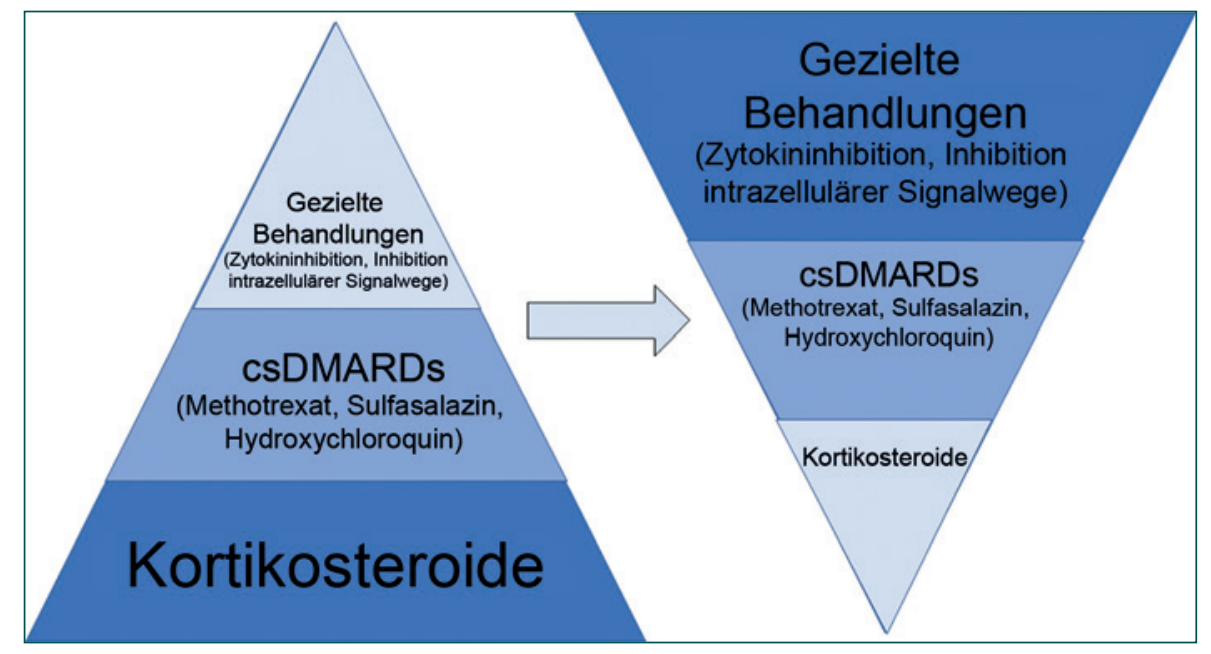

ein rekombinantes Fusionsprotein, das die extrazelluläre Domäne des humanen CTLA-4 und ein Fragment der Fc-Domäne des humanen IgG1 enthält und zur Behandlung von RA eingesetzt wird, stellt einen Sonderfall dar. Es wirkt durch Hemmung der CD28-B7-vermittelten Kostimulation von T-Zellen auf der Ebene der dendritischen Zellen und hebt so die Kostimulation von TZellen auf, die den CTLA-4- und PD-1/PL-L1-Signalwegen vorgeschaltet ist, was zu einer schnellen und globalen Zellanergie führen kann. In Anbetracht der Struktur und des Wirkmechanismus von Abatacept könnte sein Einsatz bei lebensbedrohlichen Ereignissen erwogen werden, was jedoch noch zu beweisen ist. Tatsächlich wurde in einem Fallbericht eine erfolgreiche Remission der durch Nivolumab verursachten Myokarditis gezeigt [58]. Seine Wirksamkeit und Sicherheit müssen noch in Studien nachgewiesen werden.

In den letzten Jahren ist eine neue Kategorie von DMARD erschienen: die sogenannten zielgerichteten synthetischen DMARD (tsDMARD), zu denen die Janus-Kinase (JAK)-Inhibitoren gehören (Tab. 2). Zur JAK-Familie gehören 4 Mitglieder: JAK1, JAK2, JAK3 und TYK2. Dabei handelt es sich um zytoplasmatische Tyrosinkinasen, die die intrazelluläre Signalübertragung durch Assoziation mit Typ-1- und Typ-II-Zytokinrezeptoren vermitteln [59]. Die JAK-Aktivierung führt zur Aktivierung ihrer nachgeschalteten Substrate, der STAT-Proteine (Signal Transducer and Activator of Transcription), gefolgt von deren Translokation in den Zellkern und der anschließenden Aktivierung der Zielgene [60]. IL-6 ist eines der Zytokine, die den JAK/STAT-Signalweg nutzen, um ihr intrazelluläres Signal auszusenden. Außerdem signalisieren IFN vom Typ I über JAK1 und JAK3, während IFN vom Typ II (INF- $\gamma$ ) über JAK2 signalisiert [61]. IFN- $\gamma$ ist für die Expression von PD-L1 und PD-L2 unverzichtbar und ein Marker für die Reaktion auf CPI [62]. Da der JAK/STAT-Signalweg mit der INF-Signalgebung zusammenhängt, liegt die Hypothese nahe, dass die JAK-Hemmung bei der Behandlung von CPI-bedingten unerwünschten Ereignissen, aber auch bei der Therapieresistenz eine Rolle spielen könnte. Tatsächlich haben präklinische Studien gezeigt, dass die Kombination von JAK-Hemmung und CPI eine Überwindung der CPI-Resistenz ermöglicht, möglicherweise durch eine Verringerung der Entzündung in der Tumormikroumgebung [63]. Da INF- $\gamma$ aufgrund seiner Bedeutung für die PD-L-Expression kein wünschenswertes Ziel im Rahmen der Krebsimmuntherapie ist, sollte JAK2-INF- $\gamma$ beibehalten werden. Die erste Generation von JAK-Inhibitoren wie Tofacitinib und Baricitinib wirkt auf JAK2 (Baricitinib stärker als Tofacitinib), aber die zweite Generation von JAK-Inhibitoren ist selektiver, z.B. Upadacitinib, ein selektiver JAK1-Inhibitor, der kürzlich für die Behandlung von RA zugelassen wurde. Die klinische Wirksamkeit und Sicherheit von JAK-Inhibitoren im Zusammenhang mit einem irAE muss noch nachgewiesen werden.

\section{Fazit}

Es bedarf eines besseren Verständnisses der Pathophysiologie der rheumatischen irAE sowie der Prädiktoren für die Entwicklung dieser neuen Art von unerwünschten Ereignissen. In den Behandlungsleitlinien wird weitgehend der Einsatz von Glukokortikoiden als Erstlinientherapie empfohlen, wenn die symptomatische Therapie nicht wirksam ist sowie bei anhaltender und/oder mittelschwerer/schwerer Entzündung. Zielgerichtete Therapien stehen in der Behandlungspyramide weiter oben, wenn die Reaktion auf Glukokortikoide und konventionelle, breit angelegte Immunsuppressiva unzureichend ist sowie bei schweren Formen von irAE. Die auf präklinischen Daten basierende Evidenz gibt jedoch im Hinblick auf das potenzielle Risiko einer Beeinträchtigung der antitumoralen Wirkung Anlass zur Sorge, und es besteht eine gewisse klinische Evidenz im Hinblick auf die negativen Auswirkungen einer Behandlung mit Kortikosteroiden auf die Wirksamkeit von ICI bei NSCLC. Dieses erhebliche Risiko von Glukokortikoiden sowie die hohe Wirksamkeit und die potenzielle synergistische Wirkung neuerer, gezielter Immunmodulatoren wie TNF- und IL-6-Blocker sprechen für einen Paradigmenwechsel und eine umgekehrte Behandlungspyramide, bei der csDMARD und bDMARD in der Behandlungssequenz früher in Betracht gezogen werden sollten (Abb. 3). Künftige Daten aus pro- 
spektiven Studien und randomisierten klinischen Studien, von denen einige noch laufen, werden weitere Erkenntnisse zu dieser äußerst wichtigen klinischen Frage liefern.

\section{Beiträge der Autoren}

Alle Autoren haben zur Erstellung dieser Übersicht beigetragen.

\section{Finanzierung}

Open-Access-Finanzierung durch das Karolinska-Institut.

\section{Interessenkonflikt}

KC hat Beratungshonorare von Eli Lilly, AbbVie und Pfizer erhalten. ML: keine. GT: keine. IG: keine. AC: keine.

\section{Lizenzangabe}

Chatzidionysiou K, Liapi M, Tsakonas G, Gunnarsson I, Catrina A. Treatment of rheumatic immune-related adverse events due to cancer immunotherapy with immune checkpoint inhibitors-is it time for a paradigm shift? Clin Rheumatol. 2021;40(5):1687-1695. (DOI: 10.1007/s10067-02005420-w). ${ }^{\odot} 2020$ The Author(s). (Übersetzung; Additional Information, Rights and Permissions gekürzt), lizensiert unter CC BY 4.0 (https://creativecommons.org/licenses/by/4.0/deed.de).

\section{Literatur}

Die Literatur ist unter www.karger.com/doi/10.1159/000521939 abrufbar 